\title{
Associação entre apoio social e nível de atividade física em adolescentes
}

\section{Association between social support and physical activity level in adolescents}

\section{AUTORES \\ Thiago Silva Piola ${ }^{1}$ (D) \\ Eliane Denise Araújo Bacil ${ }^{1,2}$ (D) \\ Michael Pereira da Silva ${ }^{1}$ (D) \\ Ana Beatriz Pacífico ${ }^{1}$ (D) \\ Wagner de Campos $^{1}$ (i) \\ 1 Universidade Federal do Paraná, Centro de Estudos em Atividade Física e Saúde. Curitiba, Paraná, Brasil. \\ 2 Universidade Positivo, Curitiba, Paraná, Brasil}

\section{CONTATO}

Thiago Silva Piola

tspthiago@hotmail.com

Rua Coração de Maria, 92, Jardim Botânico, Curitiba, Paraná, Brasil. CEP: 80215-370.

DOI

$10.12820 /$ rbafs.23e0021

\section{(ब) $\Theta$}

Copyright: This is an open-access article distributed under the terms of the Creative Commons Attribution License ${ }^{\circledR}$, which permits unrestricted use, distribution, and reproduction in any medium, provided that the original author and source are credited.

\begin{abstract}
RESUMO
Analisar a associação do apoio social de pais e amigos com o nível de atividade física de adolescentes. Estudo transversal, com uma amostra de 952 adolescentes $(15,72 \pm 1,48$ anos e $53,8 \%$ do sexo feminino). Foram avaliados diferentes tipos de apoio social para atividades físicas, provenientes dos pais e amigos. Um questionário foi utilizado para mensurar o nível de atividade física. $\mathrm{O}$ estado nutricional foi determinado com base no índice de massa corporal. As associações foram testadas por meio da regressão de Poisson, com variância robusta. $\mathrm{O}$ apoio social dos pais do tipo assistir a prática de atividades físicas nos adolescentes eutróficos (“às vezes" - RP = 1,60; IC95\%: 1,16-2,19 e "sempre" - RP = 1,82; IC95\%: 1,09-3,04) e com sobrepeso (“às vezes" - RP = 3,26; IC95\%: 1,23-8,63) e comentar positivamente sobre a prática de atividades físicas nos adolescentes eutróficos ("às vezes" - $\mathrm{RP}=1,49$; IC95\%: 1,08-2,07) e com sobrepeso ("às vezes"- RP = 3,11; IC95\%: 1,12-8,59) associaram-se ao nível de atividade física. $\mathrm{O}$ apoio social dos amigos do tipo praticar atividades junto nos adolescentes com sobrepeso ("sempre"- RP = 2,57; IC95\%: 1,02-6,44) e assistir a prática de atividades físicas nos adolescentes eutróficos ("sempre"- RP = 1,52; IC95\%: 1,01-2,32) e com sobrepeso ("sempre" $-\mathrm{RP}=3,68$; IC95\%: 1,19-11,35) associaram-se ao nível de atividade física. O apoio social fornecido pelos pais e amigos foi associado a maior prática de atividades físicas em adolescentes eutróficos e com sobrepeso.
\end{abstract}

Palavras-chave: Atividade motora; Apoio social; Estado nutricional.

ABSTRACT

To analyze the association of the social support of parents and friends with the physical activity level of normal weight and overweight adolescents. A cross-sectional study with a sample of 952 adolescents $(15.72 \pm$ 1.48 years, $53.8 \%$ girls). Different types of social support for physical activities from parents and friends were evaluated. A questionnaire measured physical activity level. Body mass index evaluated nutritional status. Poisson regression, with robust variance tested the associations. Parents social support was associated with attend the physical activities for normal weight adolescents ("sometimes" $-P R=1.60 ; C I 95 \%: 1.16-2.19$ and "always" $-P R=1.82$; CI95\%: 1,09-3,04) and overweight ("sometimes" $-P R=3.26, C I 95 \%: 1.23-8.63$ ) and comments positively about physical activities for normal weight adolescents ("sometimes" $-P R=1.49$; CI95\%: 1.08-2.07) and overweight ("sometimes" $-P R=3.11$; CI95\%: 1.12-8.59). Friends social support was associated for practice activities together for overweight adolescents ("always" $-P R=2.57$; CI95\%: 1.02-6.44) and attend physical activity for normal weight adolescents ("always" $-P R=1.52$; CI95\%: 1.01-2.32) and overweight ("always" - PR =3.68; CI95\%:1.19-11.35). Parents and friends social support associated with greater physical activity levels in both normal weight and overweight adolescents.

Keywords: Motor activity; Social support; Nutritional status.

\section{Introdução}

Os benefícios da prática habitual de atividade física estão bem elucidados na literatura ${ }^{1}$. No entanto, a prática insuficiente de atividade física apresenta-se como um problema frequentemente evidenciado em muitos estudos envolvendo adolescentes ${ }^{2,3}$. Diante disto, esforços vêm sendo aplicados para a identificação de correlatos e determinantes da atividade física no intuito do melhor entendimento da adoção da prática de atividade física em especial nos adolescentes, visto a importância desta fase para a adoção de hábitos que serão levados para as fases futuras da vida ${ }^{2}$.

O apoio social apresenta-se como um importante correlato da atividade física ${ }^{2}$, sendo amplamente investigado e reportado em estudos transversais ${ }^{4-6}$. Nos estudos de Duncan et al. ${ }^{6}$ é destacada a associação positiva do apoio social de amigos com o engajamento de adolescentes em atividades físicas regulares. Prado et al. ${ }^{4}$ destacaram que, além do apoio social dos amigos, a importância deste reforço por parte da família.

Um dos diversos benefícios da prática habitual de atividade física na adolescência é sua atuação na pre- 
venção de controle do sobrepeso e obesidade ${ }^{7}$. Porém, mesmo com os problemas de saúde associados a esta doença sendo bem elucidados pela literatura ${ }^{8}$, no $\mathrm{Bra}^{-}$ sil, as prevalências de sobrepeso em adolescentes apresentam valores acima de $20 \%$ para os meninos e acima de $58 \%$ para as meninas ${ }^{8}$.

Sabendo que o sobrepeso está associado a menores níveis de atividade física8 e que o apoio social está associado a maiores níveis de atividade física ${ }^{4-6}$, o presente estudo tem o objetivo de verificar a associação do apoio social de pais e amigos com o nível de atividades físicas de adolescentes, e se esta relação pode ser moderada pelo estado nutricional.

\section{Métodos}

No ano de 2014, realizou-se um estudo transversal, com uma amostra representativa de adolescentes matriculados na rede estadual de São José dos Pinhais, Paraná. O município, de porte médio, é o sexto maior do estado com uma população de 302.759 habitantes e está localizado na região metropolitana de Curitiba.

Para o cálculo amostral a priori, foi considerado uma associação de 1,49 (medida de razão de prevalên(ia) $)^{9}$ entre o apoio social e a prática de atividades físicas, uma prevalência de expostos (insuficientemente ativos) de $50 \%{ }^{3}$, um nível de confiança de $95 \%(\alpha=0,05)$ em um poder de $80 \%(\beta=0,20)$. Assim, a amostra mínima foi estimada em 327 sujeitos, com $80 \%$ de chances de rejeitar corretamente a hipótese nula, e com um acréscimo de 30\% para possíveis perdas e recusas, o tamanho amostral mínimo estimado foi de 426 adolescentes.

Para a seleção da amostra, foram estabelecidos três estágios: i) seleção de todas as escolas que ofertavam o ensino médio matutino ( $\mathrm{n}=18)$; ii) seleção aleatória simples, uma turma de cada série do ensino médio e; iii) seleção de todos os alunos destas séries participar do estudo.

Foram adotados os seguintes critérios de exclusão: adolescentes com limitações físicas e/ou cognitivas que comprometessem a prática de atividades físicas (informado pelo escolar) e ter reportado o estágio maturacional pré-púbere. Adolescentes que não entregarem o termo de consentimento livre e esclarecido assinado pelos pais ou responsáveis ou que faltou a escola no dia da coleta de dados foram considerados como perda amostral.

No total, foram avaliados 1.060 adolescentes. Para este estudo, foram excluídos os participantes com limitações físicas $(n=2)$, os pertencentes ao estágio maturacional pré-púbere $(n=26)$, além dos que responderam aos questionários de forma incorreta e/ou incompleta, faltaram no dia da coleta ou ainda se negaram a participar do estudo $(n=80)$. Assim, a amostra final do estudo foi composta por 952 adolescentes, resultando em um poder de $99,7 \%$ de chances de rejeitar corretamente a hipótese nula. A amostra também possibilita identificar razões de prevalências acima de 1,28 como risco e abaixo de 0,75 como proteção, em prevalências acima de $50 \%$ para os suficientemente ativos. O estudo foi aprovado pelo Comitê de Ética em Pesquisa da Universidade Federal do Paraná (CAAE: 36759414.0.0000.0102).

$\mathrm{O}$ apoio social para a prática de atividades físicas foi avaliado por meio um questionário validado para adolescentes brasileiros ${ }^{10}$ (consistência interna com valores de Alfa de Conbrach $-\alpha=0,87$ e coeficiente de correlação intraclasse - CCI de 0,92 , ambos no escore geral). O instrumento avalia seis tipos de apoio social fornecido por pais e amigos, sendo o apoio social recebido dos pais "estimularam”, "praticaram junto", "levaram”, "assistiram”, "comentaram” e "conversaram". Em relação aos amigos, os tipos de apoio social avaliados foram: "estimularam", "praticaram junto", "convidaram", "assistiram", "comentaram” e "conversaram”. As opções de resposta estavam em escala Likert de quatro pontos e os adolescentes reportaram a frequência semanal "nunca", "raramente", "frequentemente" e "sempre" que os pais e os amigos forneceram algum tipo de auxílio e/ou a assistência (apoio social) para a prática de atividades físicas durante uma semana normal. Para fins de análise, foram adotadas as categorias de respostas: "nunca" (nunca e raramente), "as vezes (frequentemente)" e "sempre".

A prática de atividades físicas foi reportada pelos adolescentes através do preenchimento do Self-Administered Activity Checklist ${ }^{11}$ (CCI de 0,88 e índice Kappa de 0,59 para a reprodutibilidade; correlação de Spearman de 0,62 para a validade, todos os valores de $\mathrm{p}<0,01)$. Os adolescentes reportam eram a frequência semanal e a duração na participação em 24 tipos de atividades físicas, com intensidades moderadas a vigorosas na última semana. $\mathrm{O}$ escore da atividade física foi calculado com a soma do produto da frequência semanal pelo volume, em minutos, despendido em cada atividade. E para as análises, foram considerados insuficientemente ativos os adolescentes com um volume semanal de atividades físicas menor do que 420 minutos $^{12}$.

O estado nutricional, foi determinado a partir do índice de massa corporal (IMC) - massa corporal (kg) / estatura $\left(\mathrm{m}^{2}\right)^{13}$, e classificado em "eutrófico" e "sobrepeso"14. 
Estudos prévios que analisaram fatores associados a prática de atividade física, observaram o sexo, a classe econômica e a maturação sexual como importantes variáveis explicativas da atividade física do adolescente ${ }^{2,3}$, 9. Neste estudo, estas variáveis foram tratadas como variáveis de controle: sexo (masculino e feminino); classe econômica (avaliada com base no número de utensílios domésticos na residência, presença de empregada mensalista e a escolaridade do responsável financeiro pelo domicílio, sendo categorizadas em "baixa" (classes C e D), "intermediária" (classes B1 e B2) e "elevada" (classes A1 e A2). O estágio de maturação sexual foi determinado por meio do método proposto por Tanner ${ }^{16}$, no qual os estágios maturacionais se dividem entre 1 (pré-púbere), 2, 3 e 4 (púbere) e 5 (pós-púbere). A classificação nos estágios foi autoavaliada pelos próprios adolescentes, por meio da análise da pilosidade pubiana através de imagens ${ }^{17,18}$. Os adolescentes no estágio 1 foram excluídos das análises, por ser considerada uma fase de pré-adolescência ${ }^{19}$.

A descrição das variáveis foi feita por distribuição de frequências absolutas e relativas. O teste do Qui-quadrado foi utilizado para comparar as proporções de e suas possíveis diferenças verificadas pelo teste de Qui-quadrado. A regressão de Poisson, com variância robusta, foi utilizada para testar as possíveis associações entre a frequência semanal dos diferentes tipos de apoio social recebido de pais e amigos com a prática de atividades físicas, com o estado nutricional sendo testado como possível efeito moderador na relação entre as variáveis. Após as análises bivariadas, foram realizadas análises multivariáveis considerando as variáveis estágio maturacional, sexo e classe econômica, como potenciais fatores de confusão. As variáveis entraram no modelo pelo método de "entrada forçada". Para os tipos de apoio social associados à prática de atividades físicas foram verificadas a fração atribuída populacional (FAP) a partir da razão de prevalência (RP) encontrada nas análises ajustadas, utilizando a equação $\mathrm{FAP}=\mathrm{RP}$ - 1 / RP x $100^{20}$, onde o resultado, em termos percentuais, estima a diminuição do nível de atividade física que ocorreria sem a exposição do apoio social. Todas as análises foram realizadas no pacote estatístico SPSS (IMB SPSS for Windows, 24.0; SPSS Inc.,) com um nível de significância estabelecido em $\mathrm{p} \leq 0,05$.

\section{Resultados}

A amostra final foi composta por 952 adolescentes (53,8\% de meninas), com idade média de 15,72 $\pm 1,48$ anos. A maior proporção de adolescentes pertenceu ao estágio maturacional púbere $(71,7 \%)$, foi classificada como insuficientemente ativa $(82,2 \%)$, de classe econômica intermediária (56,5\%) e eutróficos (83,5\%) - Tabela 1.

O tipo de apoio social mais comum recebido dos pais foi o estímulo para a realização de atividades físicas $(22,0 \%)$, e o menos reportado foi praticar atividades físicas em conjunto $(6,4 \%)$. Com relação ao apoio social recebido pelos amigos, praticar atividades físicas junto com o adolescente foi o mais frequente $(23,8 \%)$, enquanto o menos frequente foi comentar positivamente sobre a prática de atividades físicas (12,8\%) - Tabela 1 .

Na Tabela 2, estão os resultados da análise bivariada para a associação entre componentes de apoio social e a atividade física dos adolescentes. $\mathrm{O}$ apoio do tipo "assistiram você praticando atividades físicas" "às vezes" - RP = 1,59; IC95\%: 1,15-2,18 e "sempre" - RP = 1,83; IC95\%: 1,09-3,06) e "comentaram positivamente sobre a sua prática de atividades físicas" ("às vezes" - $\mathrm{RP}=$ 1,51; IC95\%: 1,09-2,10) fornecido pelos pais se associou a atividade física nos adolescentes eutróficos. Em relação ao apoio fornecido pelos amigos, foram observadas associações positivas para "Assistiram você praticando atividades físicas" "“às vezes"- $\mathrm{RP}=1,51$; IC95\%: 1,00-2,88) para os adolescentes eutróficos (Tabela 2).

$\mathrm{Na}$ análise ajustada, o apoio social recebido dos pais, foi associado positivamente para os componentes "assistiram você praticando atividades físicas” nos adolescentes eutróficos ("às vezes"- RP = 1,60; IC95\%: 1,162,19 e "sempre" - RP = 1,82; IC95\%: 1,09-3,04) e para os adolescentes com sobrepeso (“às vezes" $-\mathrm{RP}=3,26$; IC95\%: 1,23-8,63). Associações positivas também observadas para o apoio do tipo "comentaram positivamente sobre sua prática de atividades físicas" ("às vezes” - RP = 1,49; IC95\%: 1,08-2,07) para os adolescentes eutróficos e para os adolescentes com sobrepeso $(\mathrm{RP}=$ 3,11; IC95\%: 1,12-8,59) - Tabela 3.

$\mathrm{O}$ apoio social dos amigos esteve ainda associado a prática de atividades físicas dos adolescentes com sobrepeso para o apoio do tipo "praticaram as atividades com você" ("sempre" - RP = 2,57; IC95\%: 1,02-6,44), além do apoio do tipo "assistiram você praticando atividades físicas” que se mostrou associado a prática de atividades físicas de adolescentes eutróficos ("sempre"$\mathrm{RP}=1,52$; IC95\%: 1,01-2,32) e com sobrepeso ("sempre" - RP = 3,68; IC95\%: 1,19-11,35).

A partir das análises ajustadas, as associações do apoio social dos pais para a prática de atividades físicas dos adolescentes eutróficos e com sobrepeso, res- 
Tabela 1 - Decrição das variáveis estado nutricional, estágio maturacional, sexo, prática de atividades físicas, classe econômica e apoio social dos adolescentes de São José dos Pinhais, Paraná (n = 952).

\begin{tabular}{|c|c|c|c|c|c|c|c|}
\hline \multirow{3}{*}{ Variável } & \multicolumn{6}{|c|}{ Estado nutricional } & \multirow{3}{*}{$\mathrm{p}$} \\
\hline & \multicolumn{2}{|c|}{ Todos } & \multicolumn{2}{|c|}{ Eutrófico } & \multicolumn{2}{|c|}{ Sobrepeso } & \\
\hline & $\mathrm{n}$ & $\%$ & $\mathrm{n}$ & $\%$ & $\mathrm{n}$ & $\%$ & \\
\hline \multicolumn{8}{|l|}{ Estado nutricional } \\
\hline Eutrófico & - & - & 795 & 83,5 & - & - & \\
\hline Sobrepeso & - & - & - & - & 157 & 16,5 & \\
\hline \multicolumn{8}{|l|}{ Estágio maturacional } \\
\hline Púbere & 683 & 71,7 & 571 & 71,8 & 112 & 71,3 & 0,972 \\
\hline Pós-púbere & 269 & 28,3 & 224 & 28,2 & 45 & 28,7 & \\
\hline \multicolumn{8}{|l|}{ Sexo } \\
\hline Masculino & 440 & 46,2 & 375 & 47,2 & 65 & 41,4 & 0,210 \\
\hline Feminino & 512 & 53,8 & 420 & 52,8 & 92 & 58,6 & \\
\hline \multicolumn{8}{|l|}{ Prática de atividades físicas } \\
\hline Insuficientemente ativo & 783 & 82,2 & 649 & 81,6 & 134 & 85,4 & 0,311 \\
\hline Suficientemente ativo & 169 & 17,8 & 146 & 18,4 & 23 & 14,6 & \\
\hline \multicolumn{8}{|l|}{ Classe econômica } \\
\hline Baixa & 278 & 29,2 & 113 & 14,2 & 22 & 14,0 & 0,550 \\
\hline Intermediária & 538 & 56,6 & 453 & 57,1 & 85 & 54,1 & \\
\hline Elevada & 135 & 14,2 & 228 & 28,7 & 50 & 31,8 & \\
\hline \multicolumn{8}{|l|}{ Apoio social recebido dos pais } \\
\hline \multicolumn{8}{|c|}{ Estimularam você a praticar atividades físicas } \\
\hline Nunca & 127 & 13,4 & 104 & 13,1 & 23 & 14,7 & 0,812 \\
\hline Às vezes & 615 & 64,7 & 520 & 65,4 & 95 & 60,9 & \\
\hline Sempre & 209 & 21,9 & 171 & 21,5 & 38 & 24,4 & \\
\hline \multicolumn{8}{|c|}{ Praticaram atividades físicas com você } \\
\hline Nunca & 421 & 44,3 & 355 & 44,7 & 66 & 42,3 & 0,388 \\
\hline Às vezes & 469 & 49,3 & 392 & 49,3 & 77 & 49,4 & \\
\hline Sempre & 61 & 6,4 & 48 & 6,0 & 13 & 8,3 & \\
\hline \multicolumn{8}{|c|}{ Levaram você para praticar atividades físicas } \\
\hline Nunca & 381 & 40,1 & 320 & 40,3 & 61 & 39,1 & 0,787 \\
\hline Às vezes & 392 & 41,2 & 327 & 41,1 & 65 & 41,7 & \\
\hline Sempre & 178 & 18,7 & 148 & 18,6 & 30 & 19,2 & \\
\hline \multicolumn{8}{|c|}{ Assistiram você praticando atividades físicas } \\
\hline Nunca & 455 & 47,9 & 382 & 48,1 & 73 & 46,8 & 0,880 \\
\hline Às vezes & 430 & 45,3 & 357 & 45,0 & 73 & 46,8 & \\
\hline Sempre & 65 & 6,8 & 55 & 6,9 & 10 & 6,4 & \\
\hline \multicolumn{8}{|c|}{$\begin{array}{l}\text { Comentaram positivamente sobre sua pratica de } \\
\text { atividades físicas }\end{array}$} \\
\hline Nunca & 393 & 41,4 & 341 & 42,9 & 52 & 33,3 & 0,063 \\
\hline Às vezes & 419 & 44,1 & 340 & 42,8 & 79 & 50,6 & \\
\hline Sempre & 138 & 14,5 & 113 & 14,2 & 25 & 16,1 & \\
\hline \multicolumn{8}{|c|}{$\begin{array}{l}\text { Conversaram com você sobre sua prática de } \\
\text { atividades físicas }\end{array}$} \\
\hline Nunca & 335 & 35,2 & 284 & 35,7 & 51 & 32,7 & 0,471 \\
\hline Às vezes & 473 & 49,7 & 393 & 49,4 & 80 & 51,3 & \\
\hline Sempre & 143 & 15,1 & 118 & 14,8 & 25 & 16,0 & \\
\hline \multicolumn{8}{|l|}{ Apoio social recebido dos amigos } \\
\hline \multicolumn{8}{|c|}{ Estimularam você a praticar atividades físicas } \\
\hline Nunca & 312 & 32,8 & 253 & 31,8 & 59 & 37,8 & 0,281 \\
\hline Às vezes & 464 & 48,8 & 395 & 49,7 & 69 & 44,2 & \\
\hline Sempre & 175 & 18,4 & 147 & 18,5 & 28 & 17,9 & \\
\hline
\end{tabular}

Continua... 
... continua

\begin{tabular}{|c|c|c|c|c|c|c|c|}
\hline \multirow{3}{*}{ Variável } & \multicolumn{6}{|c|}{ Estado nutricional } & \multirow{3}{*}{$\mathrm{p}$} \\
\hline & \multicolumn{2}{|c|}{ Todos } & \multicolumn{2}{|c|}{ Eutrófico } & \multicolumn{2}{|c|}{ Sobrepeso } & \\
\hline & $\mathrm{n}$ & $\%$ & $\mathrm{n}$ & $\%$ & $\mathrm{n}$ & $\%$ & \\
\hline \multicolumn{8}{|c|}{ Praticaram atividades físicas com você } \\
\hline Nunca & 262 & 27,5 & 213 & 26,8 & 49 & 31,4 & 0,611 \\
\hline Às vezes & 463 & 48,7 & 395 & 49,7 & 68 & 43,6 & \\
\hline Sempre & 226 & 23,8 & 187 & 23,5 & 39 & 25,0 & \\
\hline \multicolumn{8}{|c|}{ Convidaram você para praticar atividades físicas } \\
\hline Nunca & 213 & 22,4 & 167 & 21,0 & 46 & 29,5 & $0,041^{*}$ \\
\hline Às vezes & 517 & 54,4 & 439 & 55,2 & 78 & 50,0 & \\
\hline Sempre & 221 & 23,2 & 189 & 23,8 & 32 & 20,5 & \\
\hline \multicolumn{8}{|c|}{ Assistiram você praticando atividades físicas } \\
\hline Nunca & 382 & 40,2 & 322 & 40,5 & 60 & 38,5 & 0,730 \\
\hline Às vezes & 435 & 45,7 & 361 & 45,4 & 74 & 47,4 & \\
\hline Sempre & 134 & 14,1 & 112 & 14,1 & 22 & 14,1 & \\
\hline \multicolumn{8}{|c|}{$\begin{array}{l}\text { Comentaram positivamente sobre sua prática de } \\
\text { atividades física }\end{array}$} \\
\hline Nunca & 406 & 42,7 & 337 & 42,4 & 69 & 44,2 & 0,837 \\
\hline Às vezes & 423 & 44,5 & 360 & 45,3 & 63 & 40,4 & \\
\hline Sempre & 122 & 12,8 & 98 & 12,3 & 24 & 15,4 & \\
\hline \multicolumn{8}{|c|}{$\begin{array}{l}\text { Conversaram com você sobre sua prática de } \\
\text { atividades físicas }\end{array}$} \\
\hline Nunca & 269 & 28,3 & 213 & 26,8 & 56 & 35,9 & 0,274 \\
\hline Às vezes & 495 & 52,1 & 429 & 54,0 & 66 & 42,3 & \\
\hline Sempre & 187 & 19,7 & 153 & 19,2 & 34 & 21,8 & \\
\hline
\end{tabular}

*Teste de Qui-quadrado para tendência linear; $\mathrm{p} \leq 0,05$.

Tabela 2 - Efeito moderador do estado nutricional e fração atribuível populacional na associação entre o apoio social dos pais e dos amigos com a atividade física dos adolescentes. São José dos Pinhais, Paraná (n =952).

\begin{tabular}{|c|c|c|c|c|c|c|c|c|c|c|c|c|}
\hline & \multicolumn{12}{|c|}{ Estrado nutricional } \\
\hline & \multicolumn{6}{|c|}{ Eutrófico } & \multicolumn{6}{|c|}{ Sobrepeso } \\
\hline & $\mathrm{n}$ & $\%$ & $\mathrm{RP}$ & $\mathrm{IC} 95 \%$ & $\begin{array}{l}\text { FAP } \\
(\%)\end{array}$ & $\mathrm{p}$ & $\mathrm{n}$ & $\%$ & $\mathrm{RP}$ & $\mathrm{IC} 95 \%$ & $\begin{array}{c}\text { FAP } \\
(\%)\end{array}$ & $\mathrm{p}$ \\
\hline \multicolumn{13}{|c|}{ Apoio social recebido dos pais } \\
\hline \multicolumn{13}{|c|}{$\begin{array}{l}\text { Estimularam você a praticar } \\
\text { atividades físicas }\end{array}$} \\
\hline Nunca & 104 & 13,1 & 1 & - & - & - & 23 & 14,7 & 1 & - & - & - \\
\hline Às vezes & 518 & 65,4 & 0,85 & $0,56-1,31$ & $\dagger$ & 0,481 & 95 & 60,9 & 2,05 & $0,51-8,28$ & 51,22 & 0,314 \\
\hline Sempre & 170 & 21,5 & 1,02 & $0,62-1,65$ & 1,96 & 0,930 & 38 & 24,4 & 1,21 & $0,24-6,09$ & 17,36 & 0,815 \\
\hline \multicolumn{13}{|c|}{$\begin{array}{l}\text { Praticaram atividades físicas } \\
\text { com você }\end{array}$} \\
\hline Nunca & 354 & 44,6 & 1 & - & - & - & 66 & 42,3 & 1 & - & - & - \\
\hline Às vezes & 391 & 49,3 & 1,08 & $0,79-1,47$ & 7,41 & 0,615 & 77 & 49,4 & 1,59 & $0,67-3,75$ & 37,11 & 0,285 \\
\hline Sempre & 48 & 6,1 & 1,45 & $0,84-2,49$ & 31,03 & 0,179 & 13 & 8,3 & 2,17 & $0,64-7,33$ & 53,92 & 0,218 \\
\hline \multicolumn{13}{|c|}{$\begin{array}{l}\text { Levaram você para praticar } \\
\text { atividades físicas }\end{array}$} \\
\hline Nunca & 319 & 40,2 & 1 & - & - & - & 61 & 39,1 & 1 & - & - & - \\
\hline Às vezes & 327 & 41,2 & 0,99 & $0,71-1,37$ & $\dagger$ & 0,961 & 65 & 41,7 & 1,40 & $0,61-3,20$ & 28,57 & 0,419 \\
\hline Sempre & 147 & 18,5 & 1,08 & $0,72-1,62$ & 7,41 & 0,691 & 30 & 19,2 & 0,76 & $0,21-2,66$ & $\dagger$ & 0,672 \\
\hline \multicolumn{13}{|c|}{$\begin{array}{l}\text { Assistiram você praticando } \\
\text { atividades físicas }\end{array}$} \\
\hline Nunca & 381 & 48,0 & 1 & - & - & - & 73 & 46,8 & 1 & - & - & - \\
\hline
\end{tabular}

Continua... 


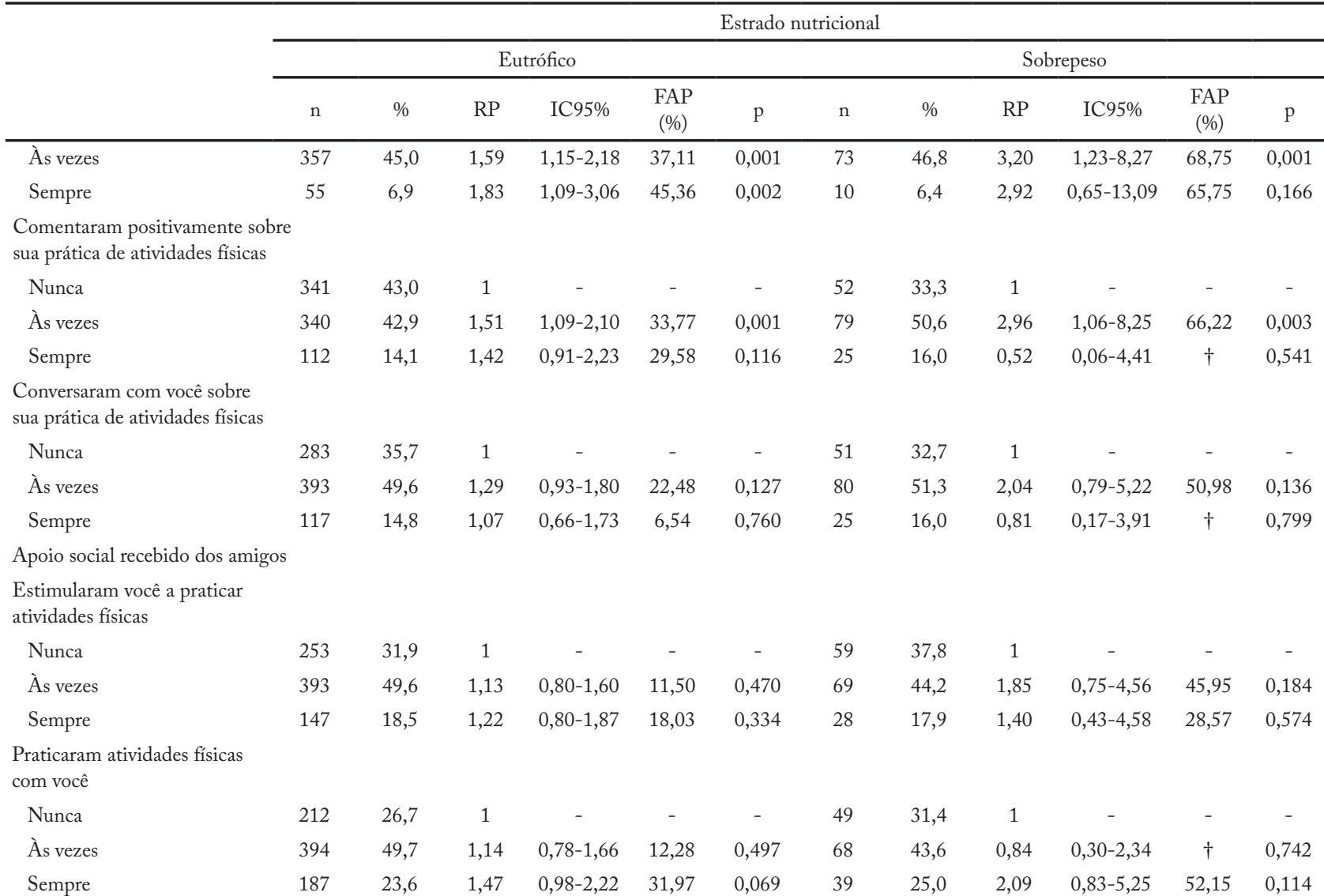

Convidaram você para praticar atividades físicas

\begin{tabular}{|c|c|c|c|c|c|c|c|c|c|c|c|c|}
\hline Nunca & 167 & 21,1 & 1 & - & - & - & 46 & 29,5 & 1 & - & - & - \\
\hline Às vezes & 437 & 55,1 & 1,19 & $0,79-1,78$ & 15,97 & 0,395 & 78 & 50,0 & 1,41 & $0,53-3,76$ & 29,08 & 0,488 \\
\hline Sempre & 189 & 23,8 & 1,32 & $0,84-2,08$ & 24,24 & 0,224 & 32 & 20,5 & 1,72 & $0,57-5,17$ & 41,86 & 0,336 \\
\hline \multicolumn{13}{|c|}{$\begin{array}{l}\text { Assistiram você praticando } \\
\text { atividades físicas }\end{array}$} \\
\hline Nunca & 320 & 40,4 & 1 & - & - & - & 60 & 38,5 & 1 & - & - & - \\
\hline Às vezes & 361 & 45,5 & 1,18 & $0,84-1,64$ & 15,25 & 0,325 & 74 & 47,4 & 2,10 & $0,79-5,58$ & 52,38 & 0,135 \\
\hline Sempre & 112 & 14,1 & 1,51 & $1,00-2,88$ & 33,77 & 0,049 & 22 & 14,1 & 2,72 & $0,87-8,52$ & 63,24 & 0,081 \\
\hline
\end{tabular}

Comentaram positivamente sobre sua prática de atividades físicas

$\begin{array}{lccccccccccccc}\text { Nunca } & 336 & 42,4 & 1 & - & - & - & 69 & 44,2 & 1 & - & - & - \\ \text { Às vezes } & 359 & 45,3 & 1,15 & 0,83-1,59 & 13,04 & 0,379 & 63 & 40,4 & 1,72 & 0,71-4,16 & 41,86 & 0,225 \\ \text { Sempre } & 98 & 12,4 & 1,43 & 0,93-2,20 & 30,07 & 0,103 & 24 & 15,4 & 2,05 & 0,71-5,86 & 51,22 & 0,175\end{array}$

Conversaram com você sobre sua prática de atividades físicas

\begin{tabular}{lcccccccccccc} 
Nunca & 213 & 26,9 & 1 & - & - & - & 56 & 35,9 & 1 & - & - & - \\
Às vezes & 427 & 53,8 & 0,92 & $0,65-1,30$ & $\dagger$ & 0,658 & 66 & 42,3 & 1,83 & $0,74-4,51$ & 45,36 & 0,181 \\
Sempre & 153 & 19,3 & 1,11 & $0,73-1,68$ & 9,90 & 0,614 & 34 & 21,8 & 1,09 & $0,33-3,61$ & 8,26 & 0,874 \\
\hline
\end{tabular}

$\mathrm{RP}=$ razão de prevalência obtida a partir da regressão de Poisson com variância robusta; IC95\% = intervalo de confiança de 95\%; FAP = fração atribuível populacional; † FAP não calculada para RP inferiores a 1,00; $\mathrm{p} \leq 0,05$.

pectivamente, indicaram FAP de 37,5 e 45,1 quando o apoio foi recebido "às vezes" e "sempre", respectivamente, e FAP de 69,3 para "assistiram você praticando atividades físicas" "às vezes". Também foi observada uma FAP de 32,8 e FAP de 67,8 para "comentaram positivamente sobre sua prática de atividades físicas", 
Tabela 3 - Efeito moderador do estado nutricional e fração atribuível populacional na associação entre o apoio social dos pais e dos amigos com a atividade física dos adolescentes, ajustados ao estágio maturacional, sexo e classe econômica. São José dos Pinhais, Paraná (n=952).

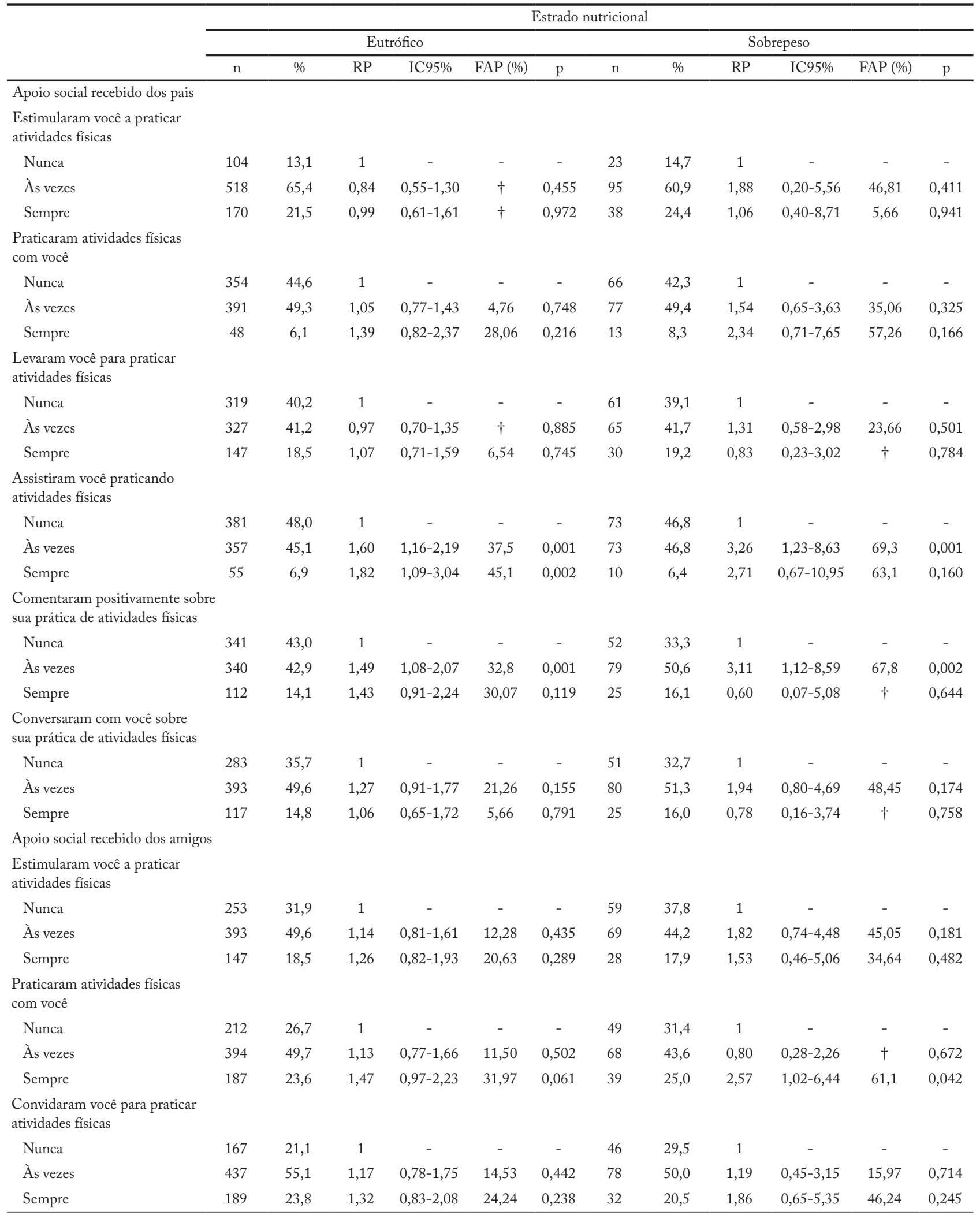

Continua... 


\begin{tabular}{|c|c|c|c|c|c|c|c|c|c|c|c|c|}
\hline & \multicolumn{12}{|c|}{ Estrado nutricional } \\
\hline & \multicolumn{6}{|c|}{ Eutrófico } & \multicolumn{6}{|c|}{ Sobrepeso } \\
\hline & $\mathrm{n}$ & $\%$ & $\mathrm{RP}$ & IC95\% & FAP $(\%)$ & $\mathrm{p}$ & $\mathrm{n}$ & $\%$ & $\mathrm{RP}$ & IC95\% & FAP (\%) & $\mathrm{p}$ \\
\hline \multicolumn{13}{|c|}{$\begin{array}{l}\text { Assistiram você praticando } \\
\text { atividades físicas }\end{array}$} \\
\hline Nunca & 320 & 40,4 & 1 & - & - & - & 60 & 38,5 & 1 & - & - & - \\
\hline Às vezes & 361 & 45,5 & 1,17 & $0,84-1,63$ & 14,53 & 0,338 & 74 & 47,4 & 2,20 & $0,81-5,96$ & 54,55 & 0,126 \\
\hline Sempre & 112 & 14,1 & 1,52 & $1,01-2,32$ & 34,2 & 0,043 & 22 & 14,1 & 3,68 & $1,19-11,35$ & 72,8 & 0,021 \\
\hline \multicolumn{13}{|c|}{$\begin{array}{l}\text { Comentaram positivamente } \\
\text { sobre sua prática de atividades } \\
\text { físicas }\end{array}$} \\
\hline Nunca & 336 & 42,4 & 1 & - & - & - & 69 & 44,2 & 1 & - & - & - \\
\hline Às vezes & 359 & 45,3 & 1,16 & $0,84-1,60$ & 13,79 & 0,369 & 63 & 40,4 & 1,8 & $0,73-4,40$ & 44,44 & 0,194 \\
\hline Sempre & 98 & 12,4 & 1,47 & $0,95-2,29$ & 31,97 & 0,084 & 24 & 15,4 & 2,3 & $0,77-6,86$ & 56,52 & 0,134 \\
\hline \multicolumn{13}{|c|}{$\begin{array}{l}\text { Conversaram com você sobre } \\
\text { sua prática de atividades físicas }\end{array}$} \\
\hline Nunca & 213 & 26,9 & 1 & - & - & - & 56 & 35,9 & 1 & - & - & - \\
\hline Às vezes & 427 & 53,8 & 0,93 & $0,65-1,31$ & $\dagger$ & 0,688 & 66 & 42,3 & 1,96 & $0,78-4,89$ & 48,98 & 0,146 \\
\hline Sempre & 153 & 19,3 & 1,12 & $0,73-1,70$ & 10,71 & 0,591 & 34 & 21,8 & 1,31 & $0,37-4,57$ & 23,66 & 0,667 \\
\hline
\end{tabular}

ambos nas frequências de "às vezes".

$\mathrm{O}$ apoio social dos amigos para adolescentes com sobrepeso apresentou uma FAP de 61,1 quando os amigos praticaram atividades em conjunto "sempre". E uma FAP de 34,2 e 72,8 para os adolescentes eutróficos e com sobrepeso quando o reforço foi do tipo "assistiram você praticando atividades físicas" sempre foi percebido.

\section{Discussão}

As análises do estudo indicaram que diferentes tipos de apoio social podem ser associados a prática de atividades físicas de adolescentes e que, esta relação pode ser moderada pelo estado nutricional, com as maiores magnitudes de associação observadas para os adolescentes com sobrepeso.

Em relação ao apoio social dos pais, este apresentou-se como um importante fator para a prática de atividades físicas, pois, assistir ao filho praticando atividades físicas poderia contribuir, para que tanto os adolescentes eutróficos quanto aqueles com sobrepeso praticassem mais atividades físicas regularmente. $\mathrm{O}$ que confirma a hipótese de que existe uma associação positiva entre a prática de atividades físicas por parte dos filhos quando seus pais assistem a esta ${ }^{5,21,22}$. Outro ponto é o papel moderador do estado nutricional observado para esta relação, indicando que esforços na promoção da saúde devem considerar este aspecto.

Os pais comentarem positivamente sobre a prática de atividades físicas é apresentado pela literatura como o tipo de suporte mais relacionado à atividade física de seus filhos ${ }^{4,6,21,22}$, porém, em todos os estudos, as análises foram moderadas pelo sexo. Todavia, sugere-se que o reforço positivo seja considerado em intervenções, uma vez que a valorização por parte de pessoas próximas tende a aumentar a autoeficácia4 que é associada a maiores níveis de atividades físicas em adolescentes ${ }^{23}$. Outro ponto é que os comentários positivos por parte dos pais poderiam aumentar a prática de atividades físicas dos adolescentes eutróficos e com sobrepeso e, considerando que a atividade física está associada positivamente a alterações no estado nutricional ${ }^{7}$, deixa claro a relevância deste reforço positivo para adolescentes eutróficos e em condições de sobrepeso.

Sobre o apoio social dos amigos, praticar atividades físicas em conjunto esteve associado a prática de atividades físicas dos adolescentes com sobrepeso, o que confirma resultados prévios que indicaram que adolescentes tendem a ter seu nível de atividade física associado ao de seus amigos ${ }^{24}$ tanto para cumprir ou deixar de cumprir as recomendações para a prática de atividades físicas ${ }^{25}$. Deixando clara a importância de se considerar práticas em grupo, pois estas facilitariam o engajamento ativo de adolescentes.

Quando amigos assistem a prática de atividades físicas, a chance de se cumprir as recomendações aumenta para adolescentes eutróficos e sobrepeso, sendo a maior magnitude da associação observada para os com sobrepeso. Este resultado mostra a importância deste 
feedback, pois amigos influenciam tanto para iniciar uma prática quanto para a permanência nesta ${ }^{25}$. Assim, o apoio social dos amigos deve ser considerado por estratégias de promoção da saúde, visto a importância que tem para a atividade física de adolescentes ${ }^{6}$, sendo ainda mais justificado pela natural independência da família e expansão das redes sociais comum nesta fase da vida ${ }^{26}$.

O presente estudo estimou a fração atribuível populacional do apoio social recebido dos pais e dos amigos na prática de atividades físicas de adolescentes e, como a grande maioria dos estudos limitou-se em verificar a associação do apoio social com a prática de atividades físicas, impossibilita comparações com outras investigações. Porém, os achados indicaram que o apoio social recebido dos pais e dos amigos pode estar associado a prática de atividades físicas de adolescentes eutróficos e com sobrepeso.

Os achados sugerem que em relação aos pais assistirem seus filhos praticando atividades físicas e comentarem positivamente sobre esta prática está positivamente associado a prática de atividades físicas de adolescentes eutróficos e com sobrepeso. E para os amigos, praticar atividades físicas em conjunto poderia elevar o nível de atividades físicas de adolescentes com sobrepeso, além de assistir o adolescente praticando atividades físicas poderia elevar os níveis de atividade física de adolescentes eutróficos e com sobrepeso.

Ainda sobre estas associações, assistir a prática de atividades físicas e comentar positivamente sobre esta, pode sugerir a hipótese de o suporte emocional ser mais relacionado a prática de atividades físicas do que o suporte instrumental ${ }^{6}$. Com isso, sugere-se que as intervenções tenham consideração especial sobre a variável apoio social, que deve ser vista como uma importante ferramenta para aumentar os níveis de atividade física em adolescentes.

Quanto as limitações do estudo, o uso de medidas autorreportadas, mesmo amplamente utilizados em estudos epidemiológicos 3 , 4,22,27, apresentam limitações quanto a acurácia e tendem a superestimar a prática de atividades físicas, assim é necessária cautela na interpretação e extrapolação dos dados.

Sobre o papel moderador do estado nutricional, é sabido que adolescentes com sobrepeso podem apresentar menor rendimento acadêmico, autoimagem negativa, depressão, exclusão social e consequentemente uma maior depressã ${ }^{28,29}$, além da preferência por atividades contrárias a atividade física ${ }^{3,30}$.

Conclui-se que o apoio social de pais e amigos pode ser associado a maiores níveis de atividade física de adolescentes eutróficos e com sobrepeso.

\section{Conflito de interesses}

Os autores declaram não haver conflito de interesses.

\section{Contribuição dos autores}

Piola TS, Bacil EDA, Silva MP e Pacífico AB participaram igualmente da concepção da ideia, coleta dos dados, análise dos dados, redação e revisão crítica do manuscrito. Campos W, participou da concepção da ideia e revisou criticamente o artigo.

\section{Referências}

1. Hallal PC, Bauman AE, Heath GW, Kohl HW, 3rd, Lee IM, Pratt M. Physical activity: more of the same is not enough. Lancet. 2012;380(9838):190-91.

2. Bauman AE, Reis RS, Sallis JF, Wells JC, Loos RJ, Martin BW. Correlates of physical activity: why are some people physically active and others not? Lancet. 2012;380(9838):258-71.

3. Barbosa Filho VC, Campos W, Bozza R, Lopes AS. The prevalence and correlates of behavioral risk factors for cardiovascular health among Southern Brazil adolescents: a cross-sectional study. BMC Pediatrics. 2012;12(130):12.

4. Prado CV, Lima AV, Fermino RC, Anez CRR, Reis RS. Social support and physical activity in adolescents from public schools: the importance of family and friends. Cad Saúde Pública. 2014;30(4):827-38.

5. Mendonca G, Cheng LA, Melo EN, Farias JuniorJC.Physical activity and social support in adolescents: a systematic review. Health Educ Res. 2014;29(5):822-39.

6. Duncan SC, Duncan TE, Strycker LA. Sources and types of social support in youth physical activity. Health Psychology. 2005;24(1):3-10.

7. Collings PJ, Wijndaele K, Corder K, Westgate K, Ridgway CL, Sharp SJ, et al. Objectively measured physical activity and longitudinal changes in adolescent body fatness: an observational cohort study. Pediatr Obes. 2016;11(2):107-14.

8. Ng M, Fleming T, Robinson M, Thomson B, Graetz N, Margono C, et al. Global, regional, and national prevalence of overweight and obesity in children and adults during 19802013: a systematic analysis for the Global Burden of Disease Study 2013. The Lancet. 2014;384(9945):5.

9. Fermino RC, Rech CR, Hino AA, Rodriguez Anez CR, Reis RS. Physical activity and associated factors in highschool adolescents in Southern Brazil. Rev Saude Publica 2010;44(6):986-95.

10. Farias Júnior JC, Lopes AS, Reis RS, Nascimento JV, Borgatto AF, Hallal PC. Development and validation of a questionnaire measuring factors associated with physical activity in adolescents. Rev Bras Saude Mater Infant. 2011;11(3):301-12.

11. Farias Júnior JC, Lopes AS, Mota J, Santos MP, Ribeiro JC, Hallal PC. Validade e reprodutibilidade de um questionário para medida de atividade física em adolescentes: uma adaptação do Self-Administered Physical Activity Checklist. Rev Bras Epidemiol. 2012;15(1):198-210.

12. WHO. Global recommendations on physical activity for health. Geneva: World Health Organization; 2010.

13. Alvarez BR, Pavan AL, Petroski E. Alturas e comprimentos 2003. 31-45 p.

14. Conde WL, Monteiro CA. Valores críticos do índice de massa corporal para classificação do estado nutricional de crianças e adolescentes brasileiros. J Pediatr (Rio J). 2006;82:266-72. 
15. Associação Brasileira de Empresas de Pesquisa - ABEP. Critério de Classificação Econômica Brasil. São Paulo: Associação Brasileira de Empresas de Pesquisa. 2013.

16. Tanner JM. Growth at adolescence: J. B. Lippincott Company; 1962.

17. Martin RHC, Uezu R, Parra A, Arena S, Bojikian L, Bohme M. Auto-avaliação da maturação sexual masculina por meio da utilização de desenhos e fotos. Rev Bras Educ Fís Esporte. 2001;15(2):212-22.

18. Bojikian LP, Massa M, Martin RHC, Teixeira CP, Kiss MAPDM, Böhme MTS. Auto-avaliação puberal feminina por meio de desenhos e fotos. Rev Bras Ativ Fis Saúde. 2012;7(2):24-34.

19. Malina RM, Rogol AD, Cumming SP, Silva MJC, Figueiredo AJ. Biological maturation of youth athletes: assessment and implications. Br J Sports Med. 2015;49(13):852-9.

20. Camey SA, Agranonik M, Radaelli J, Hirakata VN. Fração Atribuível Populacional. Rev HCPA. 2010;30(1):77-85.

21. Yao CA, Rhodes RE. Parental correlates in child and adolescent physical activity: a meta-analysis. Int J Behav Nutr Phys Act. 2015;12(10):2-38.

22. Ricardo LIC, Rombaldi A, Otte J, Perez A, Azevedo M. Associação entre apoio social e atividade física no lazer em escolares do ensino médio. Rev Bras Ativ Fis Saúde. 2013;18(2):168-76.

23. Peterson MS, Lawman HG, Wilson DK, Fairchild A, Van Horn ML. The association of self-efficacy and parent social support on physical activity in male and female adolescents. Health Psychol. 2013;32(6):666-74.

24. Seabra AF, Mendonca DM, Thomis MA, Anjos LA, Maia JA. Biological and socio-cultural determinants of physical activity in adolescents. Cad Saude Publica. 2008;24(4):721-36.
25. Pratt M, Sarmiento OL, Montes F, Ogilvie D, Marcus $\mathrm{BH}$, Perez LG, et al. The implications of megatrends in information and communication technology and transportation for changes in global physical activity. Lancet. 2012;380(9838):282-93.

26. Parra DC, Hoehner CM, Hallal PC, Ribeiro IC, Reis R, Brownson RC, et al. Perceived environmental correlates of physical activity for leisure and transportation in Curitiba, Brazil. Prev Med. 2011;52(3):4.

27. Santos SJ, Hardman CM, Barros SSH, Santos CFBF, Barros MVG. Associação entre prática de atividades físicas, participação nas aulas de Educação Física e isolamento social em adolescentes. J Pediatr (Rio J). 2015;91:543-50.

28. Risk Factor Collaboration (NCD-RisC). Worldwide trends in body-mass index, underweight, overweight, and obesity from 1975 to 2016: a pooled analysis of 2416 populationbased measurement studies in 128.9 million children, adolescents, and adults. Lancet. 2017;390(10113):2627-42.

29. Rivera JÁ, de Cossío TG, Pedraza LS, Aburto TC, Sánchez TG, Martorell R. Childhood and adolescent overweight and obesity in Latin America: a systematic review. Lancet. 2014;2(4):321-32.

30. Ferrari GLM, Matsudo V, Katzmarzyk PT, Fisberg M. Prevalence and factors associated with body mass index in children aged 9-11 years. J Pediatr. 2017;93:601-9.

Recebido: 22/12/2017

Aprovado: 04/09/2018

\section{Como citar este artigo:}

Piola TS, Bacil EDA, Silva MP, Pacifico AB, Campos W. Impacto do apoio social no nível de atividade física de adolescentes eutróficos e com sobrepeso. Rev Bras Ativ Fis Saúde. 2018;23:e0021. DOI: 10.12820/rbafs.23e0021 